\title{
Anomalous LO Phonon Lifetime in AIAs
}

\author{
M. Canonico, C. Poweleit, and J. Menéndez \\ Department of Physics and Astronomy, Arizona State University, Box 871504, Tempe, Arizona 85287-1504
}

A. Debernardi

Istituto Nazionale per la Fisica della Materia (INFM), Unitá di ricerca de Trieste Universitá e SISSA, Dipartimento Fisica Teorica, Strada Costiera 11, 34014 Trieste, Italy

S. R. Johnson and Y.-H. Zhang

Department of Electrical Engineering, Arizona State University, Box 875706, Tempe, Arizona 85287-5706

(Received 20 November 2001; published 14 May 2002)

\begin{abstract}
The temperature dependence of the frequencies and linewidths of the Raman-active longitudinal optical (LO) phonons in GaAs and AlAs have been measured. The low-temperature lifetime of the LO phonon in AlAs is found to be $9.7 \mathrm{ps}$, very close to the corresponding GaAs value of $9.5 \mathrm{ps}$. This contradicts early theoretical predictions. The agreement between theory and experiment can be restored when the accidental degeneracy between the AlAs LO phonon frequency and a feature in the two-phonon density of states is taken into account.
\end{abstract}

DOI: $10.1103 /$ PhysRevLett.88.215502

PACS numbers: $63.20 . \mathrm{Kr}, 78.30 . \mathrm{Fs}$

The lifetime of the Raman-active longitudinal optical (LO) phonon in III-V semiconductor compounds is of critical importance for the coupled dynamics of carriers and phonons in these materials. Accordingly, an intense effort has been devoted to the study of this quantity for the past 20 years [1]. The recent availability of $a b$ initio predictions of phonon lifetimes [2-5] represents a significant breakthrough, since they pinpoint the relevant LO phonon decay channels that may open the door for the engineering of phonon lifetimes.

In spite of the technological relevance of the Al-Ga-As system, the lifetime $\tau$ of LO phonons in AlAs has not been reported thus far. From time-domain measurements on an $\mathrm{Al}_{0.3} \mathrm{Ga}_{0.7}$ As alloy sample, Tsen and Morkoç find $\tau=$ $7 \pm 1 \mathrm{ps}($ at $10 \mathrm{~K})$ for both the GaAs-like and AlAs-like LO vibrations [6]. These results are quite puzzling since, as pointed out by Kash and Tsang [1], the phonon dispersion curves for GaAs and AlAs (which determine their possible LO decay channels) are quite different. A comparison of these curves for several semiconductors suggests that $\tau^{\mathrm{AlAs}}$ should be significantly longer than $\tau^{\mathrm{GaAs}}$. The first $a b$ initio estimates of phonon lifetimes added to the puzzle [3], since they predict $\tau^{\mathrm{AlAs}}=12.7 \mathrm{ps}>\tau^{\mathrm{GaAs}}=$ $9.2 \mathrm{ps}$, in agreement with Kash and Tsang's qualitative argument. The reliability of the theoretical prediction was underscored by the good agreement with the existing experimental data for GaAs [7-11].

The above discrepancies between theory and experiment could be dismissed by arguing that the GaAs-like and AlAs-like LO vibrations in AlGaAs alloys are not equivalent to the LO phonons in binary GaAs and AlAs crystals, so that a direct comparison is not legitimate. In this Letter, however, we report Raman measurements of the LO phonon frequencies and lifetimes in GaAs and AlAs. Our results show that the low-temperature lifetimes of LO phonons in GaAs and AlAs are indeed very similar. But we also find that these experimental results do not imply a failure of the $a b$ initio method. Instead, they arise from a breakdown - in the case of AlAs - of the standard formalism used to compare theory with experiment. This breakdown is caused by the accidental degeneracy between the frequency of the AlAs LO phonon and a Van Hove singularity in the two-phonon density of states. Such degeneracies have been proposed in the past - most notably in the case of the transverse-optic phonon in $\mathrm{GaP}$ - to explain highly distorted Raman line shapes $[12,13]$. The remarkable feature of our measurements is that, within experimental error, the Raman line shape of the LO phonon in AlAs remains Lorentzian, so that the anomalous aspects of the phonon decay can be unraveled only by detailed theoretical calculations.

We measured the frequency and the linewidth of the LO phonon Raman peaks as a function of temperature. Good quality AlAs samples are available only as thin epitaxial layers on GaAs substrates. This makes it very difficult to perform reliable Raman linewidth measurements. Our studies were made possible by the availability of a $2 \mathrm{~m}$ double monochromator (SOPRA). When combined with charge-coupled device detection, this instrument makes it possible to measure Raman spectra from thin layers with a resolution as good as $0.01 \mathrm{~cm}^{-1}$. Our AlAs sample was a 0.5 micron layer grown by molecular beam epitaxy on a GaAs substrate and capped with a $50 \AA \mathrm{GaAs}$ layer for protection. For the Raman measurements, we used the $488 \mathrm{~nm}$ line from an $\mathrm{Ar}^{+}$laser in the backscattering geometry. The samples were placed in a continuous flow cryostat and the temperature was varied between 10 and $300 \mathrm{~K}$. To minimize laser heating, the laser power was lowered and the monochromator's slits were opened until the instrument broadening reached a value of $0.15 \mathrm{~cm}^{-1}$, 
about $1 / 4$ of the intrinsic linewidth. This allowed us to perform all measurements with power levels below $10 \mathrm{~mW}$.

Figures 1 and 2 (insets) show typical Raman spectra. For both materials, the Raman line shape can be fit with a standard Voigt profile. The Lorentzian component of the profile is written as

$$
I(\Omega)=\Gamma_{R}(T) /\left\{\left[\Omega_{R}(T)-\Omega\right]^{2}+\Gamma_{R}^{2}(T)\right\},
$$

and it is assumed to represent the intrinsic line shape of the Raman line. The Gaussian component represents the instrumental broadening. The width of the Gaussian is set equal to the width of the laser line, and it is kept fixed for the fit of the Raman line [14]. Hence, aside from an overall amplitude, our fits include two adjustable parameters: the peak position, $\Omega_{R}(T)$, and the Lorentzian width, $\Gamma_{R}(T)=1 /(2 \tau)$.

The full width at half maximum [FWHM $=2 \Gamma_{R}(T)$ ] and line shifts obtained from our line shape fits are plotted in Figs. 1 and 2 as a function of temperature. In GaAs, the LO Raman peak is broadened by isotopic disorder. This broadening was found to be $0.12 \mathrm{~cm}^{-1}$ at low temperature from measurements of isotopically pure samples [15]. The isotopic disorder contribution has been subtracted from our experimental GaAs linewidth. No isotopic disorder is present in AlAs. By averaging data below $50 \mathrm{~K}$ $(\mathrm{GaAs})$, or $70 \mathrm{~K}(\mathrm{AlAs})$, we estimate $2 \Gamma_{R}(0)=0.56 \pm$ $0.01 \mathrm{~cm}^{-1}$ for GaAs and $2 \Gamma_{R}(0)=0.55 \pm 0.02 \mathrm{~cm}^{-1}$ for AlAs. These values correspond to lifetimes of 9.5 and $9.7 \mathrm{ps}$, respectively, confirming the apparent disagreement between experiment and the $a b$ initio results of Ref. [3] for the case of AlAs.

The existence of a discrepancy between the theoretical and the experimental low-temperature Raman linewidth for AlAs is troubling because the predicted relative AlAs/ GaAs value appears to be in error. This is surprising when one considers the similarity between the interatomic force constants for the two materials [16] and the excellent agreement between theoretical and experimental harmonic properties [17-20]. Thus, we are led to a critical review of the standard approximations used to compute phonon lifetimes in tetrahedral semiconductors. For these materials, it is assumed that the phonon self-energy (i.e., the physical quantity that describes its interaction with other phonons) can be obtained from a perturbative expansion [21] based on the quasiharmonic model, which consists in expanding the interatomic potential in powers of the displacements from the equilibrium configuration at temperature $T$. When the width of the peak is small compared with the frequency of its maximum, the Raman line shape is given, to an excellent approximation, by [22]

$$
\begin{gathered}
I(\Omega) \propto \Gamma_{\mathrm{LO}}(T, \Omega) /\left\{\left[\omega_{\mathrm{LO}}^{T}+\Delta_{\mathrm{LO}}(T, \Omega)-\Omega\right]^{2}\right. \\
\left.+\Gamma_{\mathrm{LO}}(T, \Omega)^{2}\right\} .
\end{gathered}
$$

Here $\omega_{\mathrm{LO}}^{T}$ is the quasiharmonic frequency of the Raman mode at temperature $T$, whereas $\Delta_{\mathrm{LO}}(T, \Omega)$ and $\Gamma_{\mathrm{LO}}(T, \Omega)$ are, respectively, the real and imaginary part of the phonon self-energy. To lowest order in the expansion

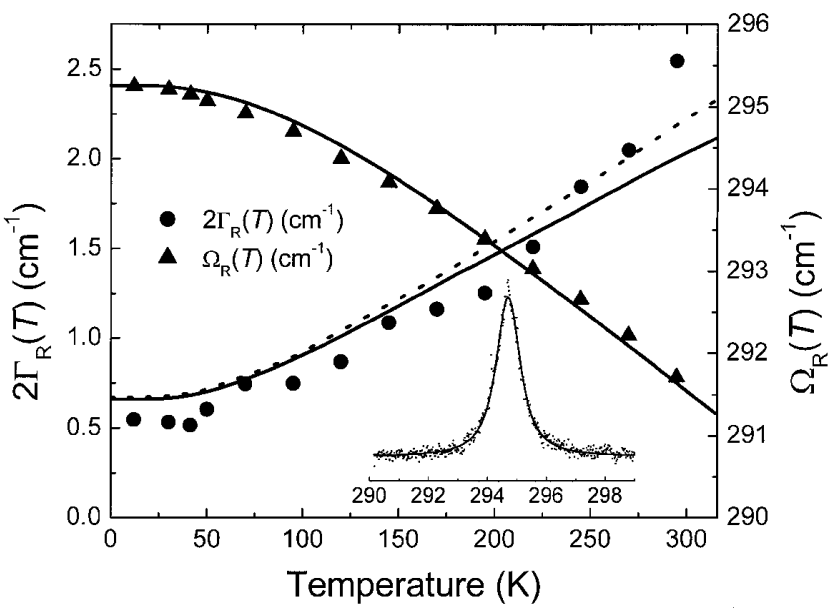

FIG. 1. The inset shows a typical GaAs LO phonon Raman spectrum obtained at $95 \mathrm{~K}$ and its Voigt profile fit. Circles (triangles) represent the Raman linewidth (line shift). The broadening due to isotopic disorder has been subtracted from the data. The errors in the Voigt fit parameters are typically smaller than the symbol size. The dashed line is a calculation of the linewidth based on Eq. (5b), which ignores the anharmonic shift of the LO phonon. The solid lines are predictions based on Eqs. (4a) and (4b), as discussed in the text. The theoretical frequency curve was rigidly upshifted by $9.3 \mathrm{~cm}^{-1}$ to match the lowest temperature experimental frequency.

of the potential, the imaginary part of the self-energy is given by [23]

$$
\begin{aligned}
\Gamma_{\mathrm{LO}}(T, \Omega)= & \frac{18 \pi^{2}}{\hbar^{2}} \sum_{\mathbf{q} j_{1} j_{2}}\left|V_{3}^{T}\left(\mathrm{LO}, \mathbf{q} j_{1},-\mathbf{q} j_{2}\right)\right|^{2} \\
& \times\left(n_{1}+n_{2}+1\right) \delta\left(\omega_{1}^{T}+\omega_{2}^{T}-\Omega\right),
\end{aligned}
$$

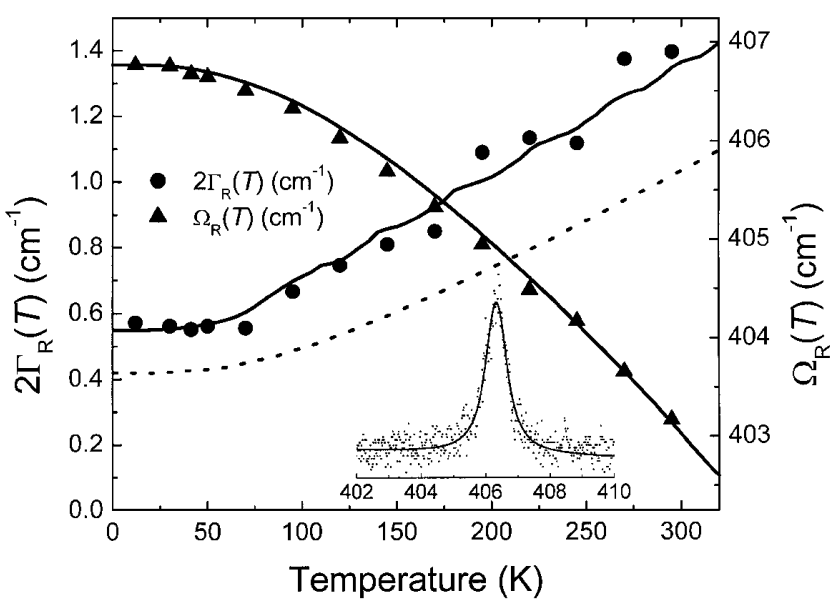

FIG. 2. The inset shows a typical AlAs LO phonon Raman spectrum obtained at $95 \mathrm{~K}$ and its Voigt profile fit. Circles (triangles) represent the Raman linewidth (line shift). The errors in the Voigt fit parameters are typically smaller than the symbol size. The dashed line is a calculation of the linewidth based on Eq. (5b), which ignores the anharmonic shift of the LO phonon. The solid lines are predictions based on Eqs. (4a) and (4b), as discussed in the text. The theoretical frequency curve was rigidly upshifted by $15.8 \mathrm{~cm}^{-1}$ to match the lowest temperature experimental frequency. 
where the sum is over phonon wave vectors and branches, $n_{i}=\left[\exp \left(\hbar \omega_{i}^{T} / k_{B} T\right)-1\right]^{-1}$ is the phonon occupation number, and the matrix element $V_{3}^{T}$ is essentially a Fourier transform of the third spatial derivative of the interatomic potential evaluated at the equilibrium position at temperature $T$ [21,23]. The lowest-order expansion of $\Delta_{\mathrm{LO}}(T, \Omega)$ includes third and fourth order anharmonic terms $[5,21,23]$. The real and imaginary parts of the selfenergy are Hilbert transforms of each other, and this relationship is used for the calculation of the real part [5]. Equation (3) corresponds to the decay of a zone center phonon of frequency $\Omega$ into pairs of phonons $\omega_{1}(\mathbf{q})$ and $\omega_{2}(-\mathbf{q})$, with the decay probability being proportional to the two-phonon density of states at the LO frequency times a (squared) anharmonic matrix element and a thermal factor.

In practical evaluations of Eq. (3), including those to be discussed here, the quantities $V_{3}^{T}, \omega_{1}^{T}$, and $\omega_{2}^{T}$ are replaced by $V_{3}^{M}, \omega_{1}^{M}$, and $\omega_{2}^{M}$, which are computed from potential derivatives at the minimum of the interatomic potential (frequencies calculated in this manner are often referred to as "bare harmonic frequencies" in the literature). Within this approximation, the $T$ dependence of the self-energy is entirely due to the explicit $T$ dependence of the occupation numbers for the decay products. For the AlAs LO phonon, the ab initio calculations indicate that the main decay channel consists of combinations of LA phonons near the $L$ point of the Brillouin zone [3].

The line shape functions in Eqs. (1) and (2) are not equivalent due to the frequency dependence of the phonon self-energy. Hence, extreme care must be exercised when comparing theory with experiment. If the self-energy can be modeled as a linear function of the frequency over the width of the Raman peak, a fit of Eq. (2) with an expression proportional to Eq. (1) gives, to an excellent approximation,

$$
\begin{aligned}
\Omega_{R}(T) & =\omega_{\mathrm{LO}}^{T}+\Delta_{\mathrm{LO}}\left[T, \Omega_{R}(T)\right], \\
\Gamma_{R}(T) & =\frac{\Gamma_{\mathrm{LO}}\left[T, \Omega_{R}(T)\right]}{\left(1+\Gamma_{1}^{2}\right)\left(1-\Delta_{1}\right)},
\end{aligned}
$$

where $\Delta_{1}\left(\Gamma_{1}\right)$ is the first derivative of the real (imaginary) part of the self-energy with respect to the frequency $\Omega$, evaluated at $\Omega=\Omega_{R}(T)$.

If the self-energy can be assumed to be essentially constant over a frequency range comparable to the anharmonic shift of the LO phonon (about $6 \mathrm{~cm}^{-1}$ for AlAs at room temperature), Eqs. (4a)(4b) can be simplified to

$$
\begin{aligned}
\Omega_{R}(T) & =\omega_{\mathrm{LO}}^{T}+\Delta_{\mathrm{LO}}\left[T, \omega_{\mathrm{LO}}^{M}\right], \\
\Gamma_{R}(T) & =\Gamma_{\mathrm{LO}}\left[T, \omega_{\mathrm{LO}}^{M}\right],
\end{aligned}
$$

where $\omega_{\mathrm{LO}}^{M}$ is the bare harmonic frequency of the LO phonon. When Eqs. (5) are inserted into Eq. (2), one recovers a Lorentzian line shape. Therefore, it is not surprising that the observation of Lorentzian line shapes is implicitly interpreted in the literature as justifying the validity of Eqs. (5). On the theoretical side, Eq. (5b) is very convenient because it does not require a calculation of the real part of the self-energy. These facts explain the widespread use of Eq. (5b) in the experimental and theoretical literature [3]. We will now show, however, that in the case of AlAs Eq. (5b) is a poor approximation, even though the Raman line shape remains Lorentzian to within experimental error. The failure of Eq. (5b) is not due to the neglect of the denominators in Eq. (4b) (which for the AlAs LO phonon tend to cancel each other and represent a correction of the order of $1 \%$ ) but to the different values of $\Gamma_{\mathrm{LO}}(T, \Omega)$ at $\Omega=\Omega_{R}(T)$ and $\Omega=\omega_{\mathrm{LO}}^{M}$.

Our calculations are based on the ab initio approach developed by Debernardi and co-workers [2-5]. The interatomic force constants are computed within the zero field anharmonic approximation [3] up to fourth order in the perturbative expansion, following the prescriptions of Refs. [3,5]. Other computational details are the same as in Ref. [5]. Figures 3 and 4 show the LO phonon self-energy for GaAs and AlAs, respectively, and the corresponding Raman line shape based on Eq. (2). The predicted temperature-dependent line shifts and linewidths can be extracted directly from these computed line shapes, and we find that for both materials these values agree with those predicted using Eqs. (4a) and (4b), the latter with the denominator set equal to unity. These predictions are also shown in Figs. 1 and 2 as solid lines, and the agreement with experiment is found to be excellent. However, the dashed lines in Figs. 1 and 2, based on Eq. (5b), represent a good approximation only for GaAs, in spite of the fact that the predicted line shape in both materials is approximately Lorentzian.

The failure of Eq. (5b) in the case of AlAs is due to the stronger frequency dependence of its LO phonon selfenergy. In the GaAs case, the LO phonon self-energy is weakly dependent on the frequency over the range of interest, as seen in Fig. 3. In contrast, the frequency

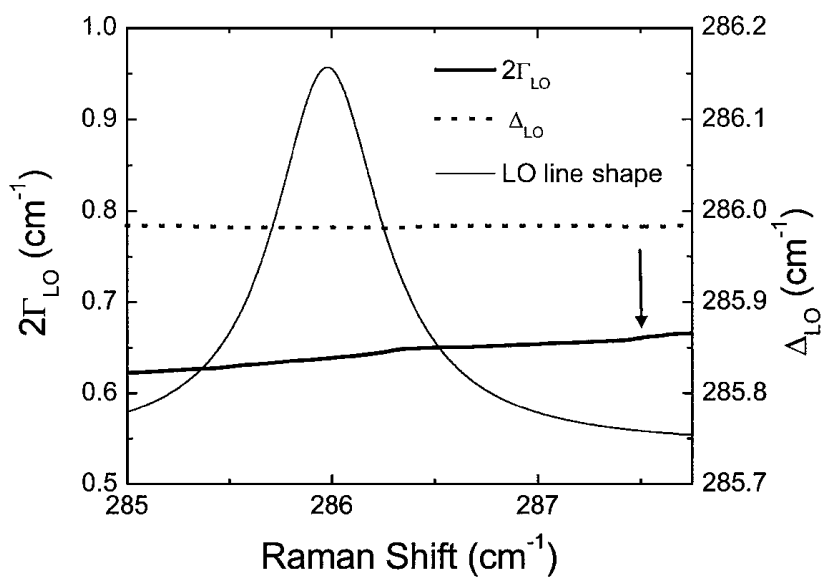

FIG. 3. The real (dotted) and imaginary (solid) parts of the LO phonon self-energy in GaAs at zero temperature. The thin solid line represents the predicted line shape using Eq. (2). The arrow marks the theoretical bare harmonic frequency of the LO mode at zero temperature. The value of $2 \Gamma_{\mathrm{LO}}$ at this frequency is the Raman linewidth according to Eq. (5b). 


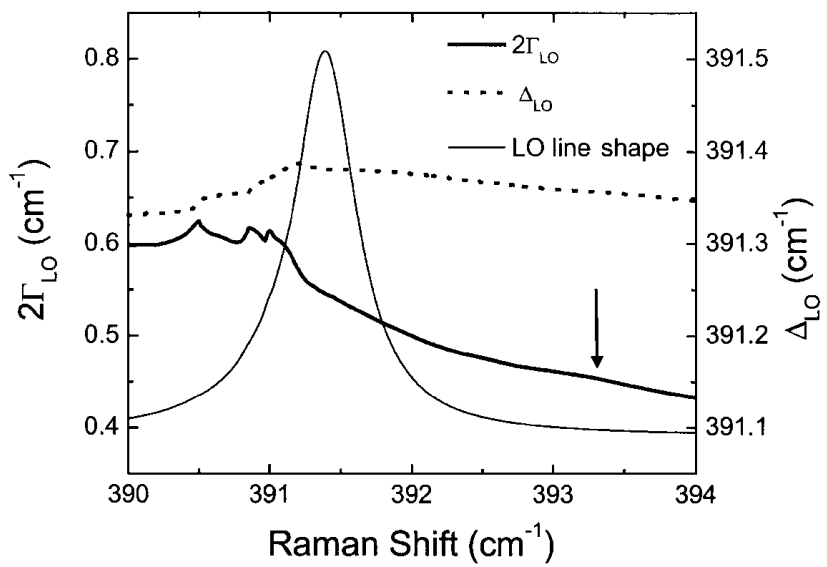

FIG. 4. The real (dotted) and imaginary (solid) parts of the phonon self-energy of the LO mode in AlAs at zero temperature. The thin solid line represents the predicted line shape using Eq. (2). The arrow marks the theoretical bare harmonic frequency of the $\mathrm{LO}$ mode at zero temperature. The value of $2 \Gamma_{\mathrm{LO}}$ at this frequency is the Raman linewidth according to Eq. (5b).

dependence of the LO phonon self-energy in AlAs, shown in Fig. 4, is much stronger. This is because the LO phonon frequency in this material is accidentally close to a local maximum in the two-phonon density of states. This local maximum - produced by decay phonons near the $L$ point of the Brillouin zone-can be seen as a cusplike feature in the calculated one-phonon density of states for AlAs [16]. Since the imaginary part of the self-energy changes significantly over a frequency range of the order of the difference between $\Omega_{R}(T)$ and $\omega_{\mathrm{LO}}^{M}$, the use of Eq. (5b) introduces sizable errors. By properly accounting for the anharmonic shift of the LO frequency, as done in Eq. (4b), the $\mathrm{LO}$ phonon is shifted even closer to the local maximum in the two-phonon density of states, and this causes a $30 \%$ increase in the linewidth value predicted from Eq. (4b) relative to the prediction from Eq. (5b).

As the temperature changes, the LO phonon frequencies move across the fine structure in the self-energy, as seen in Fig. 4, and this causes discontinuities in the first derivative of the linewidth with respect to $T$. This is the fingerprint of an anomalous decay process. Although the overall $T$ dependence of the solid line in Fig. 2 agrees with experiment, the superimposed "oscillations" are not observed experimentally (or their amplitude is smaller than the experimental error). This particular aspect of the theoretical predictions, however, should be treated with caution. The contribution from higher order terms in the perturbative expansion of the self-energy, neglected here, might be of the same order as the amplitude of the oscillations. Moreover, our calculations of the LO phonon self-energies neglect the anharmonic shifts and lifetimes of the decay products, which might affect the details of the predicted oscillations.

In summary, we have shown that the unexpectedly large LO phonon Raman linewidth in AlAs is due to the acciden- tal proximity of the LO phonon frequency to a maximum in the two-phonon density of states formed by acoustic phonons that represent the main anharmonic decay channel for the LO phonon. Were it not for this accidental degeneracy, the original linewidth calculations for AlAs [3] as well as the earlier qualitative predictions by Kash and Tsang [1] would be correct. The agreement between Tsen and Morkoç's measurements in AlGaAs alloys [6] and our results for the binary compounds is likely due to the main decay products consisting of acoustic phonons with small amplitudes on the cation sites, thereby causing these phonons to show little effect from alloy disorder. The strong dependence on small details of the phonon dispersion relations suggests that in many semiconductors it should be possible to alter the LO phonon lifetimes in appropriately engineered nanostructures.

This work was partially supported by NSF Grant No. DMR 9503904. We acknowledge the allocation of computer resources from INFM Progetto Calcolo Parallelo.

[1] J. Kash and J. Tsang, in Light Scattering in Solids VI, edited by M. Cardona and G. Günherodt (Springer-Verlag, Berlin, 1995), Vol. 68, p. 423.

[2] A. Debernardi, S. Baroni, and E. Molinari, Phys. Rev. Lett. 75, 1819 (1995).

[3] A. Debernardi, Phys. Rev. B 57, 12847 (1998).

[4] A. Debernardi and M. Cardona, Physica (Amsterdam) 263B-264B, 687 (1999).

[5] A. Debernardi, Solid State Commun. 113, 1 (2000).

[6] K. T. Tsen and H. Morkoç, Phys. Rev. B 37, 7137 (1988).

[7] D. v. d. Linde, J. Kuhl, and H. Klingenberg, Phys. Rev. Lett. 44, 1505 (1980).

[8] J. A. Kash and J. C. Tsang, Solid State Electron. 31, 419 (1988).

[9] F. Vallée and F. Bogani, Phys. Rev. B 43, 12049 (1991).

[10] F. Vallée, Phys. Rev. B 49, 2460 (1994).

[11] G. Irmer, M. Wenzel, and J. Monecke, Phys. Status Solidi (b) 195, 85 (1996).

[12] B. A. Weinstein, Solid State Commun. 20, 999 (1976).

[13] P. Galtier et al., Phys. Rev. B 28, 7334 (1983).

[14] We verified by numerical simulations that for the range of parameters used here different alternative instrumental functions (such as triangle) do not have any significant impact on the Lorentzian parameters determined from the fit.

[15] F. Widulle et al., Physica (Amsterdam) 263B-264B, 381 (1999).

[16] P. Giannozzi et al., Phys. Rev. B 43, 7231 (1991).

[17] D. Strauch and B. Dorner, J. Phys. Condens. Matter 2, 1457 (1990).

[18] E. Molinari et al., Phys. Rev. B 45, 4280 (1992).

[19] G. S. Spencer et al., Phys. Rev. B 49, 5761 (1994).

[20] J. Wagner et al., Phys. Rev. B 49, 7295 (1994).

[21] R. A. Cowley, Proc. Phys. Soc. London 84, 281 (1964).

[22] J. Menéndez and M. Cardona, Phys. Rev. B 29, 2051 (1984).

[23] M. S. Haque, Phys. Rev. B 12, 1501 (1975). 